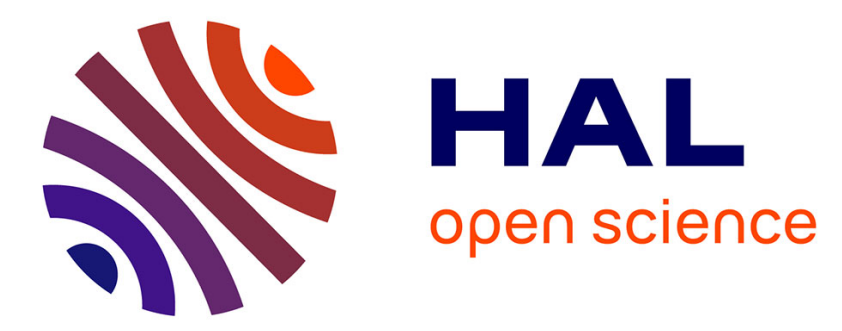

\title{
Performances of the ATLAS electromagnetic calorimeter modules
}

R. Sacco

\section{To cite this version:}

R. Sacco. Performances of the ATLAS electromagnetic calorimeter modules. ICATPP Conference on Astroparticle, Particle, Space Physics, Detectors and Medical Physics Applications 8, Oct 2003, Como, Italy. pp.1-4. in2p3-00020432

\section{HAL Id: in2p3-00020432 https://hal.in2p3.fr/in2p3-00020432}

Submitted on 20 Feb 2004

HAL is a multi-disciplinary open access archive for the deposit and dissemination of scientific research documents, whether they are published or not. The documents may come from teaching and research institutions in France or abroad, or from public or private research centers.
L'archive ouverte pluridisciplinaire HAL, est destinée au dépôt et à la diffusion de documents scientifiques de niveau recherche, publiés ou non, émanant des établissements d'enseignement et de recherche français ou étrangers, des laboratoires publics ou privés. 


\title{
Performances of the ATLAS electromagnetic calorimeter modules
}

\author{
R. $\mathrm{SACCO}^{1}$ \\ Laboratoire de l'Accélerateur Linéaire, \\ IN2P3-CNRS et Université de Paris-Sud, Bât. 200, BP 34, 91898 Orsay Cedex, France
}

\begin{abstract}
Over the last two years, four barrel and three end-cap series modules of the ATLAS electromagnetic calorimeter were exposed to electron beams of up to $245 \mathrm{GeV}$. The latest results on uniformity, linearity, energy and position resolution are presented.
\end{abstract}

\section{Introduction}

The ATLAS detector, presently under construction, will operate at the LHC proton-proton collider, aiming at searches for new physics as well as precision measurements of SM particles properties. Designed to cope with the challenges raised by a machine running at full luminosity $\left(10^{33}-10^{34} \mathrm{~cm}^{-2} \mathrm{~s}^{-1}\right), \sqrt{s}=14 \mathrm{TeV}$ and $40 \mathrm{MHz}$ bunch crossing frequency, the ATLAS electromagnetic calorimeter will play a central role in providing accurate measurements of energy, position and time of electrons and photons.

\section{Detector description}

The EM calorimeter is a lead-liquid argon ( $\ell$ Ar) sampling calorimeter with an accordion geometry that guarantees a hermetic azimuthal coverage. It is divided into two half-barrels $(|\eta|<1.475)$ and two end-caps $(1.375<|\eta|<3.2)$ and segmented in depth in three compartments. The Front sampling is made of narrow strips in $\eta$ for the precise position measurements needed in $\gamma / \pi^{0}$ separation. The Middle sampling has a depth of 16 to $18 X_{0}$ and collects most of the shower energy. The Back sampling recovers high energy tails and helps to separate hadronic from electromagnetic particles. In addition, a thin presampler corrects for energy losses in the upstream material for $|\eta|<1.8$. For a more detailed description of the detector and its construction status, see A. Jérémie's contribution to these proceedings [1].

\footnotetext{
${ }^{1}$ On behalf of the ATLAS EM group
} 


\section{Performance requirements}

The performance requirements have been defined studying the benchmark channels $H \rightarrow \gamma \gamma$, $H \rightarrow e^{+} e^{-} e^{+} e^{-}$, and heavy gauge bosons decays.

A $1 \%$ resolution on a low mass Higgs $\left(m_{H} \lesssim 150 \mathrm{GeV}\right)$ requires a sampling term below $10 \% / \sqrt{E(\mathrm{GeV})}$ and a constant term less than $0.7 \%$. The latter is the most critical issue. Thanks to a good geometric uniformity and a very precise calibration system, a $0.5 \%$ local constant term over a limited region of $\Delta \eta \times \Delta \Phi=0.2 \times 0.4$ can be achieved; a cross calibration of these different regions will be performed using $Z \rightarrow e^{+} e^{-}$to have the desired global constant term.

In order to ensure a small contribution to the $\gamma \gamma$ invariant mass resolution for low mass Higgs

search, the angular resolution on the shower direction should scale as $50 \mathrm{mrad} / \sqrt{E(G e V)}$. To ensure background rejection and new physics particle identification (decay modes with nonpointing photons), a time resolution of $100 \mathrm{ps}$ is required.

\section{Signal reconstruction and calibration}

The amplitude of the ionisation signal after shaping (having a peaking time of about $50 \mathrm{~ns}$ ) and its time are reconstructed from the linear combination of five samples, digitised at $40 \mathrm{MHz}$, located around the peak, using the optimal filtering (OF) technique [2] in order to minimise the noise. The OF coefficients are calculated from the noise autocorrelation matrix, the pulse shape and its derivative. The physics pulse shape is obtained from the calibration one, which is corrected for the difference in the input current and injection point, and for the effect of the inductance.

\section{Electron reconstruction}

The electromagnetic shower is not fully contained in a single read-out cell, therefore the energy measurement is performed, for each compartment, on a cluster of cells built around the most energetic one (e.g. $\Delta \eta \times \Delta \Phi=0.075 \times 0.075$ for the Middle compartment). To compensate for energy losses in front of the calorimeter and leakage beyond it, weights are applied to the presampler and Back compartment measurements. The finite cluster size causes a lateral leakage affecting the energy measurement with a modulation at the level of $0.4 \%$. The accordion geometry induces modulations of a similar magnitude along $\Phi$. In addition, the specific setting of the high voltage by finite sectors in the end-cap induces a linear variation of the energy response as a function of $\eta$ in each sector. The temperature of the $\ell$ Ar is stable to better than $10 \mathrm{mK}$, its variations produce a $\sim 2 \% / K$ effect. Unlike in ATLAS, the energy measurement in the test-beam is also affected at the $\pm 0.3 \%$ level by the asynchronous signal time with respect to the $40 \mathrm{MHz}$ clock. The corrections to be applied to take into account all these effects have been simulated or measured at test-beams.

\section{Test-beam results}

In 2001 and 2002, four barrel and three end-cap series modules of the ATLAS electromagnetic calorimeter were extensively tested under electron beams and using ATLAS-like (but not radiation hard) electronics. The test-beam setup and data-taking conditions are described in Refs. [3]-[4]. 
To measure the energy resolution and uniformity, energy scans from 10 to $245 \mathrm{GeV}$ have been performed at several positions. After unfolding the noise contribution, evaluated from pedestal runs, the resolution as function of the energy can be fit with the function $\sigma_{E} / E=$ $a / \sqrt{E} \oplus b$. The parameter $a$ was found to be less than $10 \%$ for each barrel module and less than $12.5 \%$ for every end-cap module. In both cases the constant term $b$ was smaller than $0.5 \%$. This is in good agreement with ATLAS specifications. To estimate the global constant term, the local constant term has to be added quadratically to the response non-uniformity. The latter has been determined with position scans for every module cell. The non-uniformity was found to be less than $0.6 \%$ for the whole barrel and end-cap modules (Fig. 1), and less than $0.5 \%$ in $\Delta \eta \times \Delta \Phi=0.2 \times 0.4$ regions, thus ensuring a global constant term below $0.7 \%$.

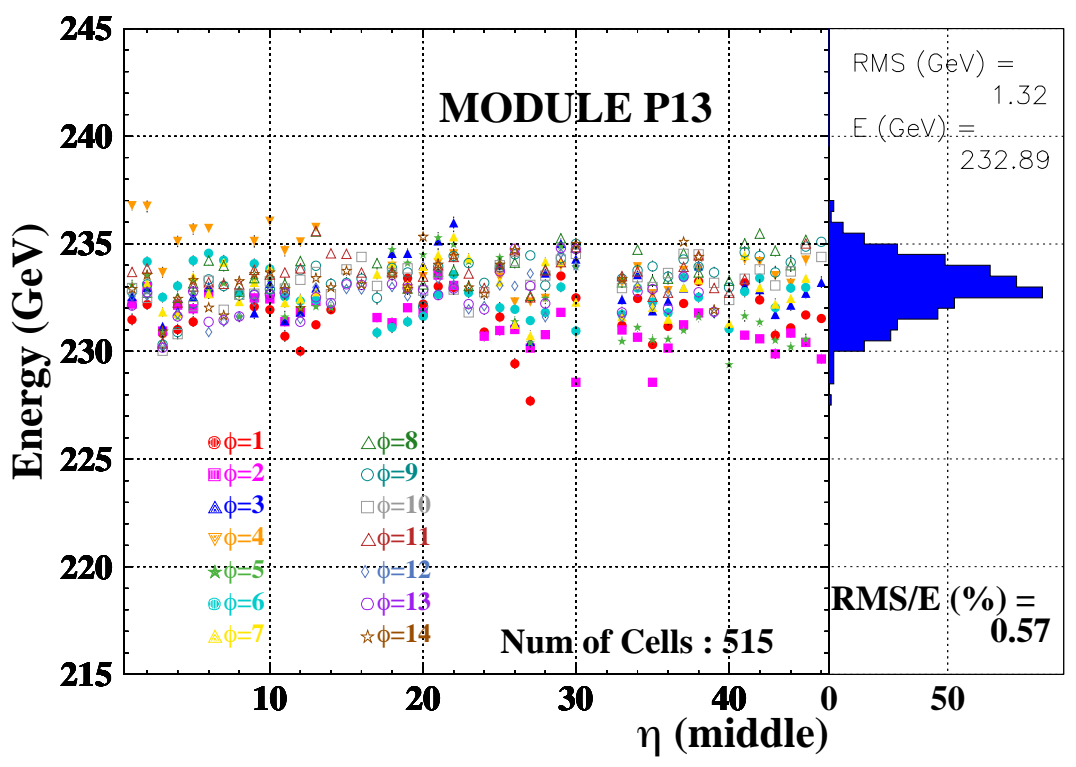

Figure 1: Response uniformity of a barrel module.

Energy weighted barycentres are calculated in the Front and Middle compartments to perform position measurements. Due to the accordion structure, only in the $\eta$ direction the bias introduced by the finite cell size (S-shape) has to be corrected. The correction is done in the Middle compartment by fitting, for each cell, the Monte Carlo predicted S-shape; in the Front compartment a method based on a logarithmic weighting of the energy in the calculation of the barycentre was preferred. The position resolution is stable across $\eta$ and amounts to about $0.15 \times 10^{-3}$ and $0.35 \times 10^{-3}$ units of pseudorapidity for the Front and Middle compartments respectively. The two separate $\eta$ measurements can be combined to reconstruct the electromagnetic particle direction. A resolution of about $53 \mathrm{mrad} / \sqrt{E}$, within ATLAS specifications, was calculated over the full test-beam energy range, as shown in Fig. 2.

To extract the intrinsic time resolution of the ATLAS modules, the distribution of the event time calculated with the OF technique was compared to the trigger time. After the subtraction of $130 \mathrm{ps}$ introduced by jitters in the trigger electronics chain (that will be reduced to about $50 \mathrm{ps}$ in ATLAS), a resolution of about 75 ps was estimated. This is well within ATLAS specifications. 


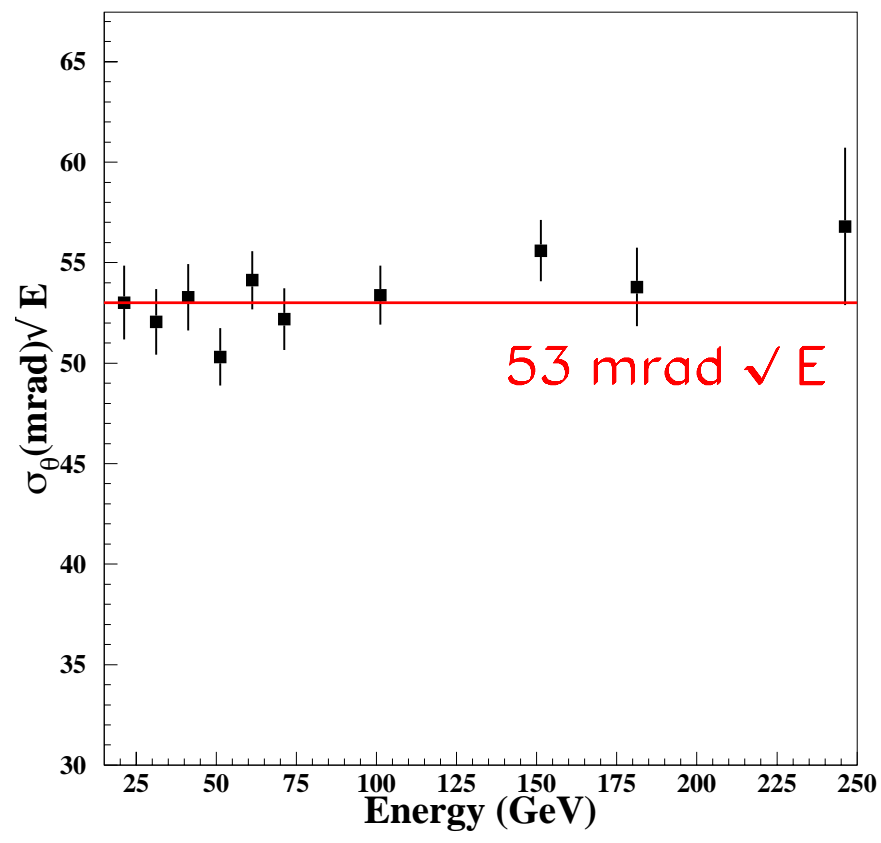

Figure 2: $\eta$ resolution as function of $\eta$, at $\phi=0.25$ for the front and middle compartments.

A big effort has been made to have a satisfactory description of the detector in the simulation. GEANT4 was used to describe the test-beam setup, and extensive comparisons with data performed. Despite the uncertainties on the amount of material upstream of the detector, a good agreement between data and simulation has been reached. In particular, the reconstructed energies in the different samplings and longitudinal and transverse profiles agree at the $1 \%$ level.

\section{Conclusions}

The analyses performed on test-beam data have shown that the series modules of the ATLAS EM calorimeter are well within the physics specifications. Further tests will be carried out in 2004 with a full barrel wedge (tracker, EM and tile calorimeters, muon detector).

\section{References}

[1] A. Jérémie, these proceedings.

[2] W.E. Cleland and E.G. Stern, Nucl. Instr. Meth. A338, 467-497 (1994).

[3] ATLAS Electromagnetic Liquid Argon Calorimeter Group, Nucl. Instr. Meth. A500, 202 (2003).

[4] ATLAS Electromagnetic Liquid Argon Calorimeter Group, Nucl. Instr. Meth. A500, 178 (2003). 